Kragujevac Journal of Mathematics

Volume 38(1) (2014), Pages 171-172.

\title{
ERRATUM TO OPTIMAL INEQUALITIES FOR EMBEDDED SPACE-TIMES
}

\author{
STEFAN HAESEN
}

\section{ERratum}

Because of the fact that the existence at a point of a semi-Riemannian manifold of an infimum (or supremum) of the sectional curvature of non-degenerate planes implies that all sectional curvatures are constant at this point [2], Definition 1 of [1] has to be replaced by the following.

Definition 1.1. For a given set of mutually orthogonal plane sections $\left\{L_{j}\right\}$ with dimensions $\left(n_{1}, \ldots, n_{k}\right)$ such that $n_{1}+\ldots+n_{k} \leq m$, the amended scalar curvature $\Lambda\left(n_{1}, \ldots, n_{k}\right)$ in the semi-Riemannian case is given by

$\Lambda\left(n_{1}, \ldots, n_{k}\right)=\tau-\left\{\sigma\left(L_{1}\right)+\ldots+\sigma\left(L_{k}\right) \mid L_{j}\right.$ a non-null plane section, $\left.L_{i} \perp L_{j}\right\}$.

With this new definition, Theorem 1 of [1] has to be replaced by the following.

Theorem 1.1. Let a m-dimensional Riemannian or Lorentzian manifold $(\mathcal{M}, g)$ be locally and isometrically embedded in a $(m+1)$-dimensional semi-Riemannian manifold $(\mathcal{N}, \widetilde{g})$ with diagonalisable Ricci tensor $\widetilde{S}$ (i.e., there exists an orthonormal basis $\left\{\vec{e}_{a}\right\}$ of $\mathcal{N}$ such that $\left.\widetilde{S}=\sum_{a=1}^{m+1} \lambda_{a} \vec{e}_{a} \otimes \vec{e}_{a}\right)$. Then, for every $k \geq 0$ and every set $\left(n_{1}, \ldots, n_{k}\right)$ such that $n_{1}<m$ and $n_{1}+\ldots+n_{k} \leq m$, we have

$$
\|H\|^{2} \geq c\left(n_{1}, \ldots, n_{k}\right) \Lambda\left(n_{1}, \ldots, n_{k}\right)-\frac{1}{2} c\left(n_{1}, \ldots, n_{k}\right)\left\{\sum_{\alpha=1}^{m} \varepsilon_{\alpha} \lambda_{\alpha}-\lambda_{m+1}\right\},
$$

if $(\operatorname{sign} \mathcal{N})=\left(s_{\mathcal{M}}+1, t_{\mathcal{M}}\right)$, and

$$
\|H\|^{2} \leq c\left(n_{1}, \ldots, n_{k}\right) \Lambda\left(n_{1}, \ldots, n_{k}\right)-\frac{1}{2} c\left(n_{1}, \ldots, n_{k}\right)\left\{\sum_{\alpha=1}^{m} \varepsilon_{\alpha} \lambda_{\alpha}+\lambda_{m+1}\right\},
$$

if $(\operatorname{sign} \mathcal{N})=\left(s_{\mathcal{M}}, t_{\mathcal{M}}+1\right)$.

Received: May 3, 2013. 
Essentially this formulation of Theorem 1 is what actually was proven in [1] and accordingly the further part of [1] still holds well.

Acknowledgment: The author thanks Luc Vrancken for bringing reference [2] to his attention.

\section{REFERENCES}

[1] S. Haesen, Optimal inequalities for embedded space-times, Kragujevac J. Math. 28 (2005), 6985.

[2] R. S. Kulkarni, The values of sectional curvatures in indefinite metrics, Comment. Math. Helvetici 54 (1979), 173-176.

Department of TeAcher EducAtion,

Thomas More Kempen University College,

LEPELSTRAat 2, B-2290 VORSElaAR,

BELGIUM

E-mail address: stefan.haesen@thomasmore.be 